\title{
The Association Between Lung Fluorodeoxyglucose Metabolism and Smoking History in 347 Healthy Adults
}

This article was published in the following Dove Press journal: Journal of Asthma and Allergy

\author{
Liang-qian Tong ${ }^{l, *}$ \\ Yan-fang Sui ${ }^{2, *}$ \\ Sheng-nan Jiang' \\ Yan-hai Yin ${ }^{3}$
}

'Department of Nuclear Medicine, Central South University Xiangya School Affiliated Haikou Hospital, Haikou, Hainan, 570208, People's Republic of China; ${ }^{2}$ Department of Rehabilitation Medicine, Central South University Xiangya School Affiliated Haikou Hospital, Haikou, Hainan, 570208, People's Republic of China; ${ }^{3}$ Department of Nuclear Medicine, Hainan Medicine College Affiliated Hainan Hospital, Haikou, Hainan, 5703II, People's

Republic of China

*These authors contributed equally to this work
Correspondence: Sheng-nan Jiang Department of Nuclear Medicine, Central South University Xiangya School Affiliated Haikou Hospital, No. 43 of Renmin Road, Meilan District, Haikou, Hainan, 570208,

People's Republic of China

$\mathrm{Tel} / \mathrm{Fax}+86089866151088$

Email jiang_ddueg@tom.com
Objective: This study aimed to evaluate the relationship between fluorodeoxyglucose metabolism and smoking history in healthy adults by analyzing lung standardized uptake value (SUV).

Methods: The ${ }^{18}$ F-fluorodeoxyglucose positron emission tomography/computed tomography $\left({ }^{18}\right.$ F-FDG PET/CT) studies of 347 patients who did not show signs of having malignant diseases or lung inflammation were retrospectively evaluated. Four circular regions of interest (ROI) were manually drawn on the upper and lower lung regions. The averages of maximum SUV (SUVmax-avr) and mean SUV (SUVmean-avr) were calculated, and the mean values of each parameter for non-smokers, ex-smokers, and current smokers were compared. The correlation between SUVmax-avr and smoking history (tobacco burden and the duration of smoking cessation) was assessed based on present smoking status. The exsmokers and current smokers were divided into three groups according to their tobacco burden, and the SUVmax-avrs of the two groups were compared.

Results: Both the mean values of SUVmax-avr and SUVmean-avr increased based on smoking history, with non-smokers having the lowest values and current smokers the highest. Tobacco burden had a positive correlation with SUVmax-avr in current smokers $(r=0.474, P<0.001)$. However, neither tobacco burden $(r=0171, P=0.162)$ nor duration of smoking cessation $(r=$ $0.212, P=0.082$ ) had a significant correlation with SUVmax-avr in ex-smokers. The mean SUVmax-avr of current smokers was significantly higher than that of ex-smokers in patients with a medium or large tobacco burden $(P=0.012, P<0.001$, respectively). Although there was no significant difference between the mean SUVmax-avrs of ex-smokers and current smokers in patients with a small tobacco burden $(P=0.888)$, the mean SUVmax-avrs of both ex-smokers and current smokers with a small tobacco burden were significantly higher than that of nonsmokers $(P<0.001, P<0.001$, respectively).

Conclusion: The findings indicate that lung SUV increases in current heavy smokers and partially decreases after the cessation of smoking, which is in line with previous reports studied by analyzingfluorodeoxyglucose (FDG) metabolism of lung specimens.

Keywords: smoking, lung, positron emission tomography/computed tomography, fluorodeoxyglucose

\section{Introduction}

Cigarette smoking is known to induce lung inflammation. ${ }^{1-4}$ It is reported that chronic inflammation of the lung tissue may result in repeated lung injury, which enhances cell turnover, potential genetic error, and epithelial-to-mesenchymal transition of the lung and ultimately leads to lung tumorigenesis. ${ }^{5}$ Parimon et $\mathrm{al}^{6}$ 
reported that patients with chronic obstructive pulmonary disease (COPD) who were treated with inhaled corticosteroids had a reduced incidence of lung cancer and death, suggesting that the inhibition of inflammation can prevent lung tumorigenicity.

${ }^{18} \mathrm{~F}$-fluorodeoxyglucose positron emission tomography/ computed tomography $\left({ }^{18} \mathrm{~F}-\mathrm{FDG} \mathrm{PET} / \mathrm{CT}\right)$ is useful in detecting and staging malignant tumors ${ }^{7-9}$ and has been used to study inflammatory disease ${ }^{10,11}$ based on the increased glucose utilization of malignant or inflammatory tissue. Although there are a few studies exploring lung standardized uptake value (SUV) in normal tissue, ${ }^{12,13}$ they focused on the differences in lung SUV based on anatomical location rather than on smoking history. As such, the relationship between lung SUV of FDG uptake and smoking history is currently unclear.

It can be theorized that lung SUV increases with the inflammatory reaction of the lung associated with smoking. Therefore, the present study assessed the differences in lung SUV based on smoking status and tobacco burden.

\section{Methods}

\section{Patients}

The ${ }^{18}$ F-FDG PET/CT studies of 502 adult patients who were referred to rule out malignancy between January 2018 and October 2019 were retrospectively analyzed. A total of 347 patients were up to the standard of the inclusion criteria and were included in the study.

Each patient's smoking history, including the duration of smoking cessation, was assessed using a validated questionnaire. ${ }^{14}$ "Pack years" means the calculative years of smoking, and was applied to measure tobacco burden. The current smokers and ex-smokers were then divided into three groups on the basis of the median tobacco burden of 15.0 pack years (for ex-smokers) and 25.0 pack years (for current smokers): (1) patients with a smaller tobacco burden $(0<$ pack years $\leq 15, \mathrm{n}=61)$; (2) patients with a medium tobacco burden $(15<$ pack years $\leq 25, \mathrm{n}=32)$; (3) patients with a larger tobacco burden (pack years $>25, \mathrm{n}=44$ ).

Inclusion criteria: (1) ${ }^{18} \mathrm{~F}-\mathrm{FDG}$ PET/CT imagings were interpreted as negative for malignancy; (2) no history of a cancer diagnosis in the last five years; (3) no history of previous chest surgery; (4) no history of exposure to environmental or occupational pollutants; (5) no evidence of inflammatory lung lesions on the unenhanced CT scanning of the ${ }^{18} \mathrm{~F}-\mathrm{FDG}$ PET/CT.

\section{${ }^{18}$ F-FDG PET/CT Imaging}

Whole-body ${ }^{18}$ F-FDG scans were acquired using a PET/CT system (Discovery STE, GE Healthcare, Milwaukee, USA) with the protocol used at our institution. All patients fasted for at least six hours before the intravenous (IV) administration of ${ }^{18}$ F-FDG by $7.4 \mathrm{MBq} / \mathrm{kg}$ of body weight. Blood glucose levels of the patients were all below $7.2 \mathrm{mmol} / \mathrm{L}$. No IV iodinated contrast was used in the last 7 days.

$\mathrm{CT}$ images were acquired a range from the skull base to the upper thigh using the following parameters: $120 \mathrm{kV}$ of peak voltage; 10 to $130 \mathrm{mAs}$ of automated tube current; 0.8 seconds of rotation time; $50 \mathrm{~cm}$ of field of view; 40 to 50 seconds of length of scan; $3.75 \mathrm{~mm}$ of slice thickness. PET data were obtained from the same anatomic locations immediately after CT acquisition, with an axial view field of $157 \mathrm{~cm}$ in the $3 \mathrm{D}$ mode at $150 \mathrm{~s} / \mathrm{bed}$ position. The CT data were used for the PET attenuation correction, and all the data of PET/CT imaging were reconstructed using a conventional iterative algorithm (ordered-subsets expectation maximization). A workstation (AW Volume Share ${ }^{\mathrm{TM}}$, GE Healthcare, USA) with multiplanar reformatted images was used for the displaying and analyzing PET/CT imagings.

\section{Image Analysis}

Circular regions of interest (ROI) about $2 \mathrm{~cm}$ in diameter on four lateral peripheral regions were manually indicated on the axial PET images simultaneously placed on both PET and CT images. Each ROI was on a single 2D axial slice: the right upper lung (RUL), right lower lung (RLL), left upper lung (LUL), and left lower lung (LLL). The ROIs of the RUL and LUL were drawn at the level of the carina, while the ROIs of the RLL and LLL were drawn at the level of the bifurcation of the intermediate bronchus (Figure 1). The maximum and mean SUVs of each ROI were obtained and defined as SUVmax-RUL, SUVmaxRLL, SUVmax-LUL, SUVmax-LLL, SUVmean-RUL, SUVmean-RLL, SUVmean-LUL, and SUVmean-LLL. The averages of SUVmax-RUL, -RLL, -LUL, and -LLL were then calculated and defined as SUVmax-avr, and the averages of SUVmean- RUL, -RLL, -LUL, and -LLL were calculated and defined as SUVmean-avr.

Body weight-normalized SUV computation was employed for the SUV algorithm. For each patient, the SUVmax $(\mathrm{g} / \mathrm{mL})$, SUVmean $(\mathrm{g} / \mathrm{mL})$, metabolic lung volume ( $\mathrm{cm} 3)$ of ROI, and total lung glycolysis $(\mathrm{g} \cdot \mathrm{cm} 3 / \mathrm{mL})$ of the ROI were measured using a standard $42 \%$ SUVmax threshold volume of interest embedding the whole ROI. 


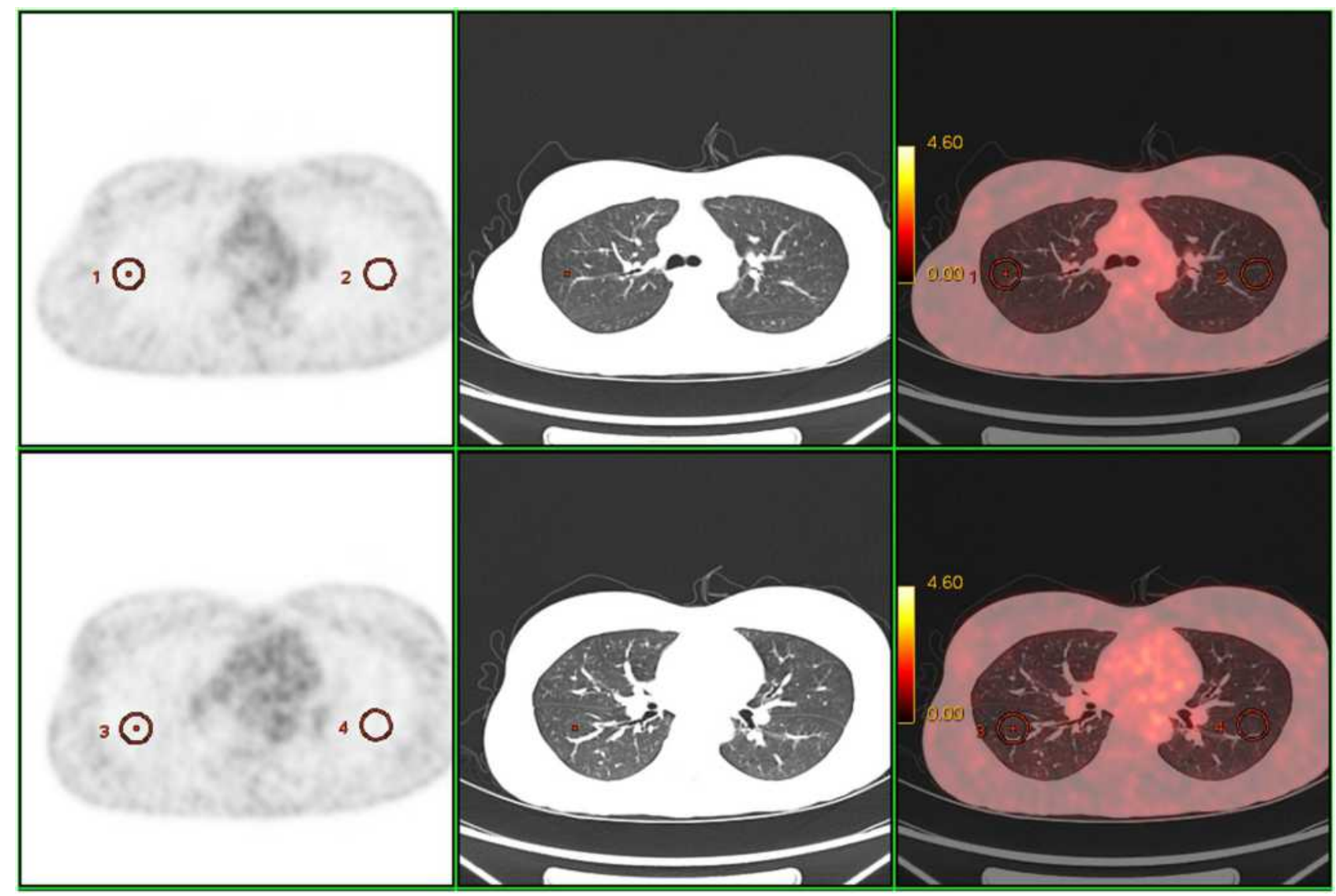

Figure I Representative ROIs of the lung on the ${ }^{18} \mathrm{~F}$-FDG PET/CT. Circular ROls were drawn on the lateral peripheral regions of the RUL, RLL, LUL, and LLL.

\section{Statistical Analysis}

To explore the influence of smoking status on lung SUV, non-smokers, ex-smokers, and current smokers were statistically analyzed using one-way analysis of variance (ANOVA) and an unpaired $t$-test. The relationship between SUVmax-avr of FDG uptake and smoking history (tobacco burden and the duration of smoking cessation) according to present smoking status was assessed using linear regression analysis. Multivariate linear regression analysis was applied in order to adjust for age. SPSS 19.0 for Windows (SPSS Inc, an IBM Company, Chicago, USA) was used for all the statistical analysis. All $P$ values were derived from two-sided tests, and $P<0$. 05 was considered statistically significant.

\section{Results}

\section{Patient Characteristics}

Table 1 shows the characteristics of the 347 patients enrolled in our study. The distribution of age ranged from 24 to 77 years (median 53.0 years). There were no significant differences in the ages among non-smokers, ex-smokers, and current smokers. While all 148 female patients were non-smokers, the 199 male patients were evenly distributed among nonsmokers $(n=62)$, ex-smokers $(n=68)$, and current smokers $(n=69)$. The median duration of smokers was higher $(25.0$ years) than ex-smokers (17.5 years); consequently, the median tobacco burden was higher in current smokers (25.0 pack years) than in ex-smokers (15.0 pack years).

\section{The Relationship Between Lung SUV and Smoking Status}

The differences in the lung SUVs of non-smokers, exsmokers, and current smokers are shown in Table 2 and Figure 2. There were significant differences in SUVmaxavr, SUVmean-avr, SUVmax-RUL, -RLL -LUL, and -LLL, and SUVmean-RLL and -LLL between non-smokers, exsmokers, and current smokers $(P<0.001)$. However, there were no significant differences in SUVmean-RUL and -LUL between non-smokers, ex-smokers, and current smokers $(P=$ $0.520, P=0.455$, respectively) (Table 2). SUVmax-RLL and -LLL and SUVmean-RLLand -LLL were significantly higher than SUVmax-RUL -LUL $(P<0.001, t$-test $)$. 
Table I Demographic Data of Non-Smokers, Ex-Smokers and Current Smokers

\begin{tabular}{|l|l|l|l|}
\hline Parameters & Non-Smokers (n=2 10) & Ex-Smokers (n=68) & Current Smokers (n=69) \\
\hline Age, years, median (range) & $53.0(24-77)$ & $54.0(36-77)$ & $51.0(38-73)$ \\
\hline Male/Female & $62 / 148$ & $68 / 0$ & $69 / 0$ \\
\hline $\begin{array}{l}\text { Smoking history } \\
\text { Packs smoked per day, median (range) } \\
\text { Smoking duration,years, median (range) } \\
\begin{array}{l}\text { Pack years, years, median (range) } \\
\text { Duration of smoking cessation, years, median (range) }\end{array}\end{array}$ & & $1.0(0.5-3.0)$ & $1.0(0.2-2.5)$ \\
\hline
\end{tabular}

Table 2 Differences in the Lung SUV According to Smoking Status

\begin{tabular}{|c|c|c|c|c|c|}
\hline Parameters & Non-Smokers $(n=210)$ & Ex-Smokers $(n=68)$ & Current Smokers $(n=69)$ & F value & $P$ value \\
\hline \multicolumn{6}{|c|}{ SUVmax, mean (SD) } \\
\hline SUVmax-avr & $0.77(0.11)$ & $0.86(0.12)$ & $1.01(0.12)$ & 114.520 & $<0.001$ \\
\hline SUVmax-RUL & $0.69(0.12)$ & $0.74(0.13)$ & $0.82(0.13)$ & 31.707 & $<0.001$ \\
\hline SUVmax-RLL & $0.85(0.13)$ & $0.98(0.12)$ & $1.24(0.12)$ & 261.068 & $<0.001$ \\
\hline SUVmax-LUL & $0.69(0.12)$ & $0.74(0.13)$ & $0.82(0.13)$ & 32.147 & $<0.001$ \\
\hline SUVmax-LLL & $0.85(0.13)$ & $0.99(0.11)$ & $1.16(0.11)$ & 169.543 & $<0.001$ \\
\hline \multicolumn{6}{|c|}{ SUVmean, mean (SD) } \\
\hline SUVmean-avr & $0.52(0.08)$ & $0.53(0.13)$ & $0.59(0.12)$ & 16.016 & $<0.001$ \\
\hline SUVmean-RUL & $0.48(0.09)$ & $0.48(0.13)$ & $0.49(0.13)$ & 0.655 & 0.520 \\
\hline SUVmean-RLL & $0.55(0.09)$ & $0.60(0.13)$ & $0.7 \mid(0.12)$ & 60.569 & $<0.001$ \\
\hline SUVmean-LUL & $0.48(0.09)$ & $0.47(0.13)$ & $0.49(0.13)$ & 0.790 & 0.455 \\
\hline SUVmean-LLL & $0.56(0.09)$ & $0.58(0.13)$ & $0.68(0.11)$ & 35.774 & $<0.001$ \\
\hline
\end{tabular}

The mean SUVmax-avr and the mean SUVmean-avr of FDG uptake progressively increased from non-smokers to ex-smokers and then to current smokers (Figure 2). The mean SUVmax-avr and the mean SUVmean-avr of current smokers were both significantly higher than those of nonsmokers $(P<0.001, P<0.001$, respectively) and ex-smokers $(P<0.001, P=0.001$, respectively). The mean SUVmax-avr and the mean SUVmean-avr of ex-smokers were significantly higher than those of non-smokers $(P<0.001, P=$ 0.035 , respectively). In non-smokers, neither the SUVmaxavr nor the SUVmean-avr showed significant differences based on sex $(P=0.892, P=0.423$, respectively).

\section{Differences in SUVmax-avr According to Smoking Status and Tobacco Burden}

The correlations between SUVmax-avr and smoking history (tobacco burden and duration of smoking cessation) in ex-smokers and current smokers are shown in Figure 3. Tobacco burden took on a positive correlation with SUVmax-avr in current smokers $(r=0.474, P<0.001)$. These differences still existed after adjustment for differences in age $(P<0.001)$. However, neither tobacco burden $(r=0.171, P=0.162)$ nor duration of smoking cessation $(r=0.212, P=0.082)$ had significant correlations with SUVmax-avr of FDG uptake in ex-smokers.

Contrasted with that of the ex-smokers, the mean SUVmax-avr of current smokers was significantly higher than that of ex-smokers in patients with a medium $(1.03 \pm$ 0.14 vs $0.88 \pm 0.16)$ or larger tobacco burden $(1.08 \pm 0.15$ vs $0.89 \pm 0.11)(P=0.012, P<0.001$, respectively $)$. However, there were no significant differences between the mean SUVmax-avr of ex-smokers $(0.91 \pm 0.13)$ and current smokers $(0.91 \pm 0.16)$ with a smaller tobacco burden $(P=0.888)$ (Figure 4$)$. The mean SUVmax-avrs of ex-smokers and current smokers with less tobacco burden were both significantly higher than that of nonsmokers $(0.78 \pm 0.13)(P<0.001, P<0.001$, respectively $)$.

\section{Discussion}

Our findings of this study show that lung SUV of FDG uptake increases from non-smokers to ex-smokers and then to current smokers and is associated with tobacco 

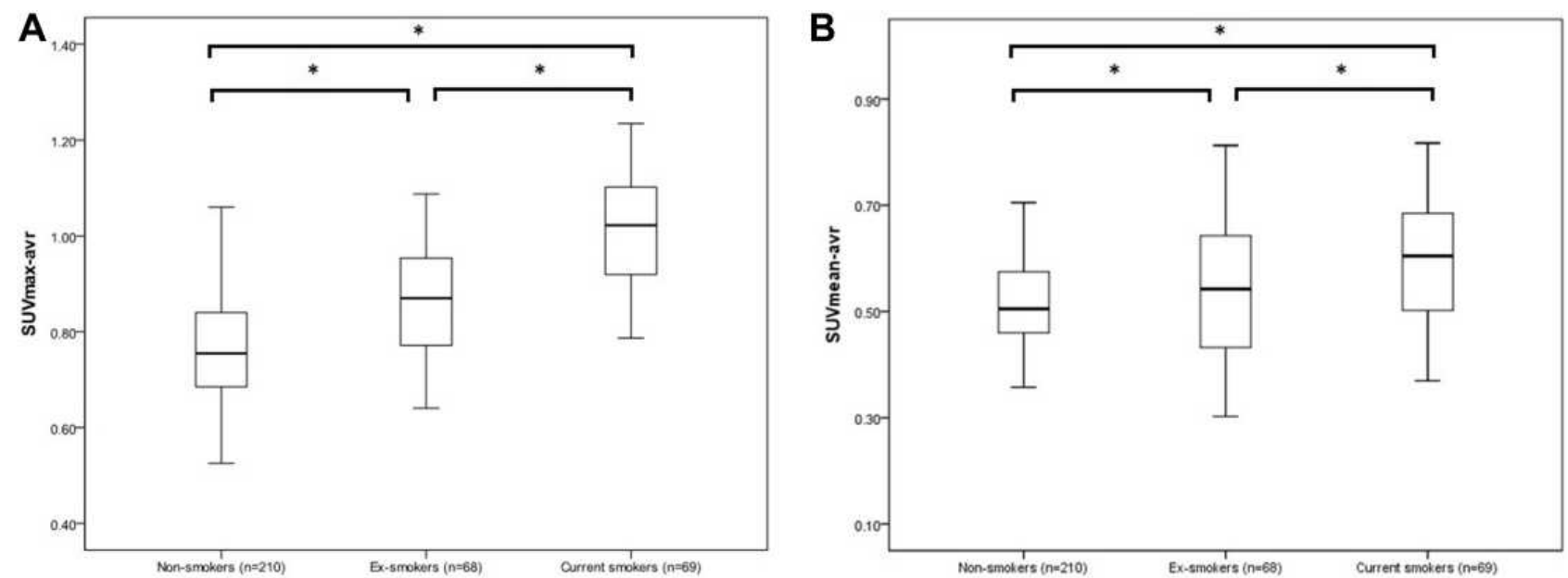

Figure 2 Differences in SUVmax-avr (A) and SUVmean-avr (B) between non-smokers, ex-smokers, and current smokers. The data are presented as box plots (median, interquartile range, range). $* P<0.05$ (two-tailed unpaired $t$-test). SUVmax-avr, the average of maximum SUVs, and SUVmean-avr, the average of mean SUVs, were measured in the RUL, RLL, LUL, and LLL.

A

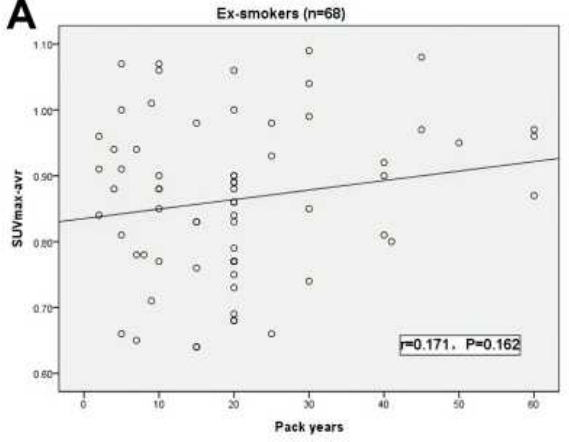

B

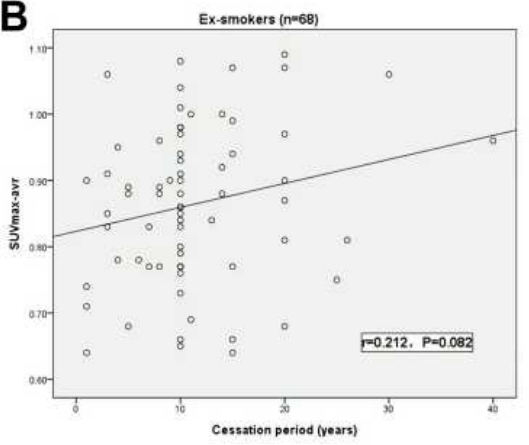

C

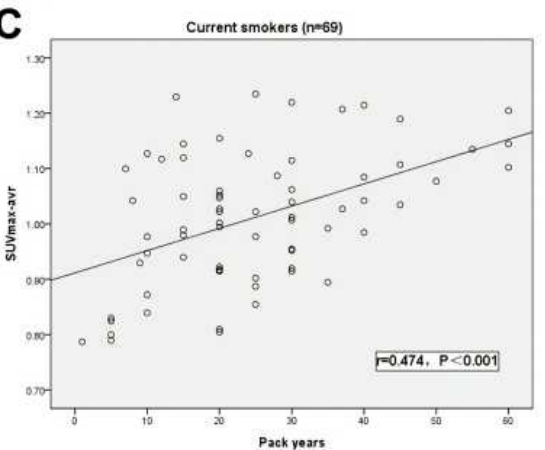

Figure 3 (A) The correlation between tobacco burden (pack years) and SUVmax-avr in ex-smokers $(r=0.171, P=0.162)$. (B) The correlation between duration of smoking cessation (years) and SUVmax-avr in ex-smokers $(r=0.212, P=0.082)$. (C) The correlation between tobacco burden (pack years) and SUVmax-avr in current smokers $(r=$ $0.474, P<0.001)$.
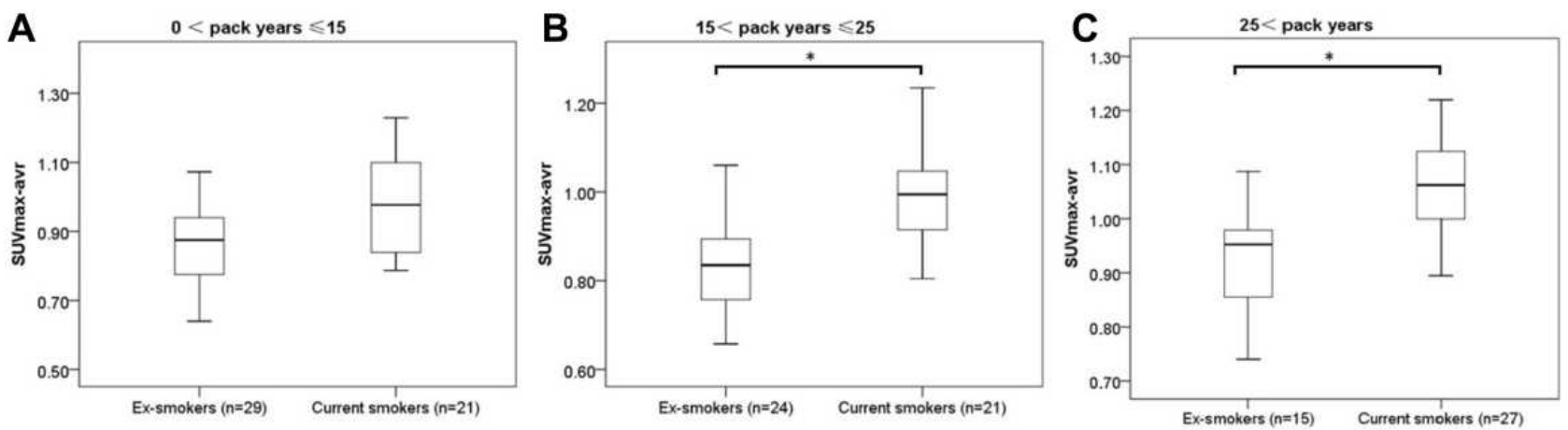

Figure 4 Differences in SUVmax-avr between ex-smokers and current smokers with a smaller tobacco burden $(0<$ pack years $\leq 15$, $(\mathbf{A})$ medium tobacco burden $(15<$ pack years $\leq 25$, (B) and larger tobacco burden (pack years $>25$, (C)). Data are presented as box plots (median, interquartile range, range). $* P<0$. 05 (two-tailed unpaired t-test). 
burden only in current smokers. No significant difference was acquired between the mean SUVmax-avrs of exsmokers and current smokers with less tobacco burden (pack years $\leq 15$ ), although they were higher than that of non-smokers.

Of the 502 patients initially studied, 49 (9.7\%) with inflammatory lung lesions on the unenhanced CT imaging of the ${ }^{18}$ F-FDG PET/CTs were excluded to remove the possibility of increased lung SUV resulting from inflammatory causes other than smoking. All ROIs were drawn on PET images corresponding to the visually normal-appearing lung regions on $\mathrm{CT}$. The average of the four different areas of ROI was calculated as a representative lung SUV. It was found that the lung SUV of FDG uptake of the lower lungs was significantly higher than that of the upper lungsincreased perfusion and vasculature of the lower lungs compared to the upper lungs may contribute to this result. ${ }^{12,13}$

Efforts were made to uniformly lace the four ROIs at the level of the carina and the bifurcation of the intermediate bronchus using a linked cursor program on ${ }^{18} \mathrm{~F}-\mathrm{FDG}$ PET/CT. These two anatomical landmarks were selected because they are easily localized on CT images.

Parimon et $\mathrm{al}^{6}$ reported that inflammation of respiratory bronchioles was identified in the surgical lung biopsy specimens of 2/24 non-smokers (8.3\%), 24/49 ex-smokers (49\%), and 83/83 current smokers (100\%). These results are in good agreement with the findings of the present study, in which it was identified that lung SUV increased step-by-step from the non-smokers to the ex-smokers and then to the current smokers. The characteristic histopathologic appearance of respiratory bronchiolitis is that the pigmented macrophages accumulated within the respiratory bronchioles and the surrounding airspaces, with minimal associated mural inflammation. ${ }^{3}$ Previous studies have suggested that macrophages contribute to fluorodeoxyglucose (FDG) accumulation in inflammatory disease. ${ }^{15}$ The accumulation of macrophages and associated inflammatory changes may be the cause of the increased lung SUV of smokers in the present study.

Although mean SUVmean-RUL and-LUL tended to increase from the non-smokers to the ex-smokers and then to the current smokers, this increase was not statistically significant. Armstrong et $\mathrm{al}^{16}$ reported that the density of normal-lung total pulmonary tissue volume (blood, extravascular water, and dry tissue volume) per total pulmonary tissue volume and gas was $0.19 \pm 0.03$. In the present study, all the ROIs were drawn on normalappearing lung regions containing gas, which may have caused the underestimation of the mean SUV of pulmonary tissue. Thus, this study demonstrated the relationship between SUVmax-avr of FDG uptake and smoking history according to present smoking status.

The smoking prevalence of the non-smokers, the exsmokers, and the current smokers in male and female patients in the present study was $31.1 \%(62 / 199) / 100.0 \%$ (148/148), 34.2\% (68/199)/0 (0/148), and 34.7\% (69/199)/ $0(0 / 148)$, respectively. In a prospective cohort study on $1,212,906$ Korean adults, the smoking prevalence of the non-smokers, the ex-smokers, and the current smokers in male and female patients was $20.1 \% / 92.1 \%, 23.0 \% / 02.5 \%$, and $57.0 \% / 5.4 \%$, respectively. ${ }^{17}$ The relatively smaller portion of smokers in the present study may be due to the inclusion criteria: it is expected that patients included in this study paid more attention to their health and smoked less than general subjects.

Kawabata et $\mathrm{al}^{18}$ reviewed patients who had received a lobectomy for lung cancer and reported that the incidence of respiratory bronchiolitis was higher in moderate smokers $(<25-\leq 50$ pack years) or heavy smokers ( $>50$ pack years) than in mild smokers ( $\leq 25$ pack years) $(33 \%$ and $34 \%$ respectively vs $9 \%$ ). These findings suggest that the positive correlation between tobacco burden and SUVmax-avr in current smokers included in the present study can be attributed to lung inflammation related to smoking.

In ex-smokers, neither tobacco burden nor duration of smoking cessation were found to be related to SUVmax-avr. Although the mean SUVmax-avr of FDG uptake of the exsmokers was significantly higher than that of the non-smokers, it was significantly lower than that of the current smokers with a medium or larger tobacco burden. This suggests that cessation of smoking, regardless of the duration, may decrease SUVmax-avr to some extent but not completely, indicating a partial recovery of the lung after inflammation. Several studies have demonstrated that airway inflammation persists

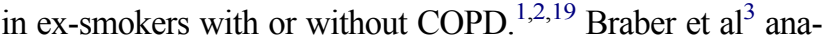
lyzed inflammatory cells and chemokine and cytokine profiles in the bronchoalveolar lavage fluid (BALF) and lung tissue of mice exposed to cigarette smoking for 20 weeks and 8 weeks after smoking cessation. Although the neutrophilic inflammation in the BALF of mice exposed to cigarette smoking was cut down after smoking cessation, inflammations of the lung tissue persisted. The elevated levels of inflammatory cytokines, such as TNF-a and IL-la, recovered to base levels after smoking cessation, but the increased IL-12 levels did not. Moreover, the levels of IL-10 - a cytokine down-regulating Th1-driven immune response-were decreased in the BALF of mice 
with exposure to cigarette smoking, and these levels were still reduced after smoking cessation in contrast to those of the control animals. The study concluded that the inflammatory changes in the lung caused by the exposure to cigarette smoking were only partially recovered after smoking cessation. The findings of the present study support this conclusion.

One of the limitations of the present study is its patient population. All the smokers included were male patients, so it was not possible to study the relationship between lung SUV and tobacco burden or smoking status in female smokers. All female patients in this study were nonsmokers, and the bias of sex may impact the significance and generalizability when conducting statistical analysis with ex-smokers and current smokers. In addition, clinical information about the history of previous airway inflammation was not available because of the study's retrospective design.

\section{Conclusion}

This study represents the first evaluation of the relationship between FDG metabolism of lung and smoking status and tobacco burden using lung SUV of FDG uptake. The data indicate that lung SUV of FDG uptake increases in the current heavy smokers and partially decreases after the cessation of cigarette smoking. These findings are in good line with previous reports analyzing FDG metabolism of lung specimens, suggesting the usefulness of ${ }^{18}$ F-FDG PET/CT for monitoring lung inflammation.

\section{Ethics Approval and Consent to Participate}

I confirm that I have read the Editorial Policy pages. This study was conducted with approval from the Ethics Committee of Central South University Xiangya School Affiliated Haikou Hospital. This study was conducted in accordance with the declaration of Helsinki. Written informed consent was obtained from all participants.

\section{Acknowledgments}

We would like to acknowledge the hard and dedicated work of all the staff that implemented the intervention and evaluation components of the study.

\section{Funding}

This study was supported by the National Natural Science Foundation of China [No. 81960556].

\section{Disclosure}

The authors declare that they have no competing interests.

\section{References}

1. Hikichi M, Mizumura K, Maruoka S, et al. Pathogenesis of chronic obstructive pulmonary disease (COPD) induced by cigarette smoke. $J$ Thorac Dis. 2019;11(Suppl 17):S2129-S2140. doi:10.21037/ jtd.2019.10.43

2. Vij N, Chandramani-Shivalingappa $P$, Van Westphal C, et al. Cigarette smoke-induced autophagy impairment accelerates lung aging, COPD-emphysema exacerbations and pathogenesis. $\mathrm{Am}$ J Physiol Cell Physiol. 2018;314(1):C73-C87. doi:10.1152/ ajpcell.00110.2016

3. Braber S, Henricks PA, Nijkamp FP, et al. Inflammatory changes in the airways of mice caused by cigarette smoke exposure are only partially reversed after smoking cessation. Respir Res. 2010;11:99. doi:10.1186/1465-9921-11-99

4. Le Y, Wang Y, Zhou L, et al. Cigarette smoke-induced HMGB1 translocation and release contribute to migration and NF-kappaB activation through inducing autophagy in lung macrophages. $J$ Cell Mol Med. 2019.

5. Pages V, Fuchs RP. How DNA lesions are turned into mutations within cells? Oncogene. 2002;21(58):8957-8966. doi:10.1038/sj. onc. 1206006

6. Parimon T, Chien JW, Bryson CL, et al. Inhaled corticosteroids and risk of lung cancer among patients with chronic obstructive pulmonary disease. Am J Respir Crit Care Med. 2007;175(7):712-719. doi:10.1164/rccm.200608-1125OC

7. Mendoza DP, Digumarthy SR. The added value of quantitative 18F-FDG-PET/CT parameters in the assessment of pulmonary lymphangitic carcinomatosis in lung cancer. J Thorac Dis. 2019;11(11): E239-E242. doi:10.21037/jtd.2019.10.10

8. Smith DE, Fernandez AJ, Da LA, et al. Accuracy of positron emission tomography and computed tomography (PET/CT) in detecting nodal metastasis according to histology of non-small cell lung cancer. Updates Surg. 2019;71(4):741-746. doi:10.1007/s13304-019-00680-x

9. Kwee TC, Basu S, Alavi A. PET/CT for unknown primary tumors. Methods Mol Biol. 2011;727:317-333.

10. Keidar Z, Gurman-Balbir A, Gaitini D, et al. Fever of unknown origin: the role of 18F-FDG PET/CT. J Nucl Med. 2008;49 (12):1980-1985. doi:10.2967/jnumed.108.054692

11. Garcia JR. Febrile syndrome of unknown origin: indications for (18) F-FDG PET/CT in inflammatory and infectious processes. Radiologia. 2017;59(3):253-263. doi:10.1016/j.rx.2016.07.007

12. Wang Y, Chiu E, Rosenberg J, et al. Standardized uptake value atlas: characterization of physiological 2-deoxy-2-[18F]fluoro-D-glucose uptake in normal tissues. Mol Imaging Biol. 2007;9(2):83-90. doi:10.1007/s11307-006-0075-y

13. Miyauchi T, Wahl RL. Regional 2-[18F]fluoro-2-deoxy-D-glucose uptake varies in normal lung. Eur J Nucl Med. 1996;23(5):517-523. doi:10.1007/BF00833385

14. Pauwels RA, Lofdahl CG, Laitinen LA, et al. Long-term treatment with inhaled budesonide in persons with mild chronic obstructive pulmonary disease who continue smoking. European Respiratory Society Study on Chronic Obstructive Pulmonary Disease. $N$ Engl J Med. 1999;340(25):1948-1953.

15. Cocker MS, Spence JD, Hammond R, et al. [18F]Fluorodeoxyglucose PET/CT imaging as a marker of carotid plaque inflammation: comparison to immunohistology and relationship to acuity of events. Int J Cardiol. 2018;271:378-386. doi:10.1016/j. ijcard.2018.05.057

16. Armstrong JD, Gluck EH, Crapo RO, et al. Lung tissue volume estimated by simultaneous radiographic and helium dilution methods. Thorax. 1982;37(9):676-679. doi:10.1136/thx.37.9.676 
17. Jee SH, Samet JM, Ohrr H, et al. Smoking and cancer risk in Korean men and women. Cancer Causes Control. 2004;15(4):341-348. doi:10.1023/B:CACO.0000027481.48153.97

18. Kawabata Y, Hoshi E, Murai K, et al. Smoking-related changes in the background lung of specimens resected for lung cancer: a semiquantitative study with correlation to postoperative course. Histopathology. 2008;53(6):707-714. doi:10.1111/j.13652559.2008.03183.x
19. Conickx G, Avila CF, van den Berge M, et al. microRNA profiling in lung tissue and bronchoalveolar lavage of cigarette smoke-exposed mice and in COPD patients: a translational approach. Sci Rep. 2017;7 (1):12871. doi:10.1038/s41598-017-13265-8

\section{Publish your work in this journal}

The Journal of Asthma and Allergy is an international, peer-reviewed open-access journal publishing original research, reports, editorials and commentaries on the following topics: Asthma; Pulmonary physiology; Asthma related clinical health; Clinical immunology and the immunological basis of disease; Pharmacological interventions and

Submit your manuscript here: https://www.dovepress.com/journal-of-asthma-and-allergy-journal new therapies. The manuscript management system is completely online and includes a very quick and fair peer-review system, which is all easy to use. Visit http://www.dovepress.com/testimonials.php to read real quotes from published authors. 